\title{
THE DETAILED MAGNETIC FIELD STRUCTURE IN THE SW QUADRANT OF M31
}

\author{
R. BECK, E.M. BERKHUIJSEN \\ Max-Planck-Institut für Radioastronomie \\ Auf dem Hügel 69 \\ 5300 Bonn 1 \\ F.R.G.
}

\begin{abstract}
A three-dimensional, arc-like structure in the magnetic field was found coming out of the plane of M31. This structure may be the first Parker-Jeans instability observed in an external galaxy.
\end{abstract}

\section{Observations and Results}

The first map of polarized radio emission of M31 made at $\lambda 11.1 \mathrm{~cm}$ with the $100-\mathrm{m}$ Effelsberg radio telescope with an angular resolution of $4: 4$ (Beck et al., 1980) was analyzed by Sofue and Takano (1981) and by Beck (1982) who found that the changes of intensity and position angle of the E-vectors with azimuthal angle are consistent with an axisymmetric, nearly toroidal magnetic field configuration. Recent $\lambda 6.3 \mathrm{~cm}$ polarization observations (Berkhuijsen et al., 1987), which are nearly free of Faraday effects, give further support to an axisymmetric field structure.

Recently we observed the total and the linearly polarized radio emission at $\lambda 20.1 \mathrm{~cm}$ from 3 fields covering the southern part of M31 with the VLA in its D-configuration. The total and polarized emission from the first two combined fields is shown in Fig. 1 with a half-power width of the synthesized beam of $75^{\prime \prime}$.

For the first field centred at $R A=00^{\text {h }} 38^{m} 36^{s}, D E C=40^{\circ} 54^{\prime}(1950.0)$ Faraday rotation measures (RM) between $\lambda 20.1 \mathrm{~cm}$ and $\lambda 6.3 \mathrm{~cm}$ were obtained by comparison with the Effelsberg data of Berkhuijsen et al. (1987) after both data sets had been smoothed to a Gaussian beamwidth of 3'. The RM ambiguity was solved with the help of the $\lambda 11.1 \mathrm{~cm}$ map of Beck et al. (1980). After correction for Faraday rotation the orientations of the magnetic field lines in the plane of the sky $\left(\chi_{B}\right)$ were obtained. The field lines are generally aligned along the dust lane outside the main optical arm.

The values of intrinsic $\mathrm{RM}\left(\mathrm{RM}_{\mathrm{i}}\right)$ only partly follow the slow variation with azimuthal angle $(\theta)$ in the plane of M31 as predicted by the axisymmetric model. Careful analysis by Beck et al. (1989) shows that the deviations are periodic with a length scale along the spiral arm of $\sim 4.5 \mathrm{kpc}$. The effect of a thermal plasma varying in electron density and/or extension along the line of sight as derived from the thermal radio emission cannot explain the observations. The periodic variations of 
the field orientation are not restricted to the plane of $\mathrm{M} 31$, but are also visible in the plane of the sky, i.e. nearly perpendicular to the plane of M31. The variation of $\chi_{B}$ is partly periodic and anticorrelated to that of $\mathrm{RM}_{i}$, i.e. the length scale along the arm is again $\sim 4.5 \mathrm{kpc}$.

The independent data of RM and $\chi_{B}$ indicate the existence of a threedimensional arc-like structure of the magnetic field on a scale of $\sim 4.5 \mathrm{kpc}$ in the plane of $\mathrm{M} 31$, superimposed onto the axisymmetric field. The scale and shape of this structure are reminiscent of the ParkerJeans instability. In such an instability the magnetic field is tied to the spiral arm at the positions of large cloud complexes and inflated out of the plane in between cloud complexes (see inset of Fig. 1).

This analysis will be continued for the other fields observed.

We thank Dr. P. Rao for reducing the data of the second field on M31.

\section{References}

Beck, R. (1982) Astron. Astrophys. 106, 121.

Beck, R., Berkhuijsen, E.M. and Wielebinski, R. (1980) Nature 283, 272.

Beck, R., Loiseau, N., Hummel, E., Berkhuijsen, E.M., Gräve, R. and Wielebinski, R. (1989) Astron. Astrophys. 222, 58.

Berkhuijsen, E.M., Beck, R. and Gräve, R. (1987) in R. Beck and R. Gräve (eds.), Interstellar Magnetic Fields, Springer, Heidelberg, p. 38.

Sofue, Y. and Takano, T. (1981) Publ. Astron. Soc. Japan 33, 47.

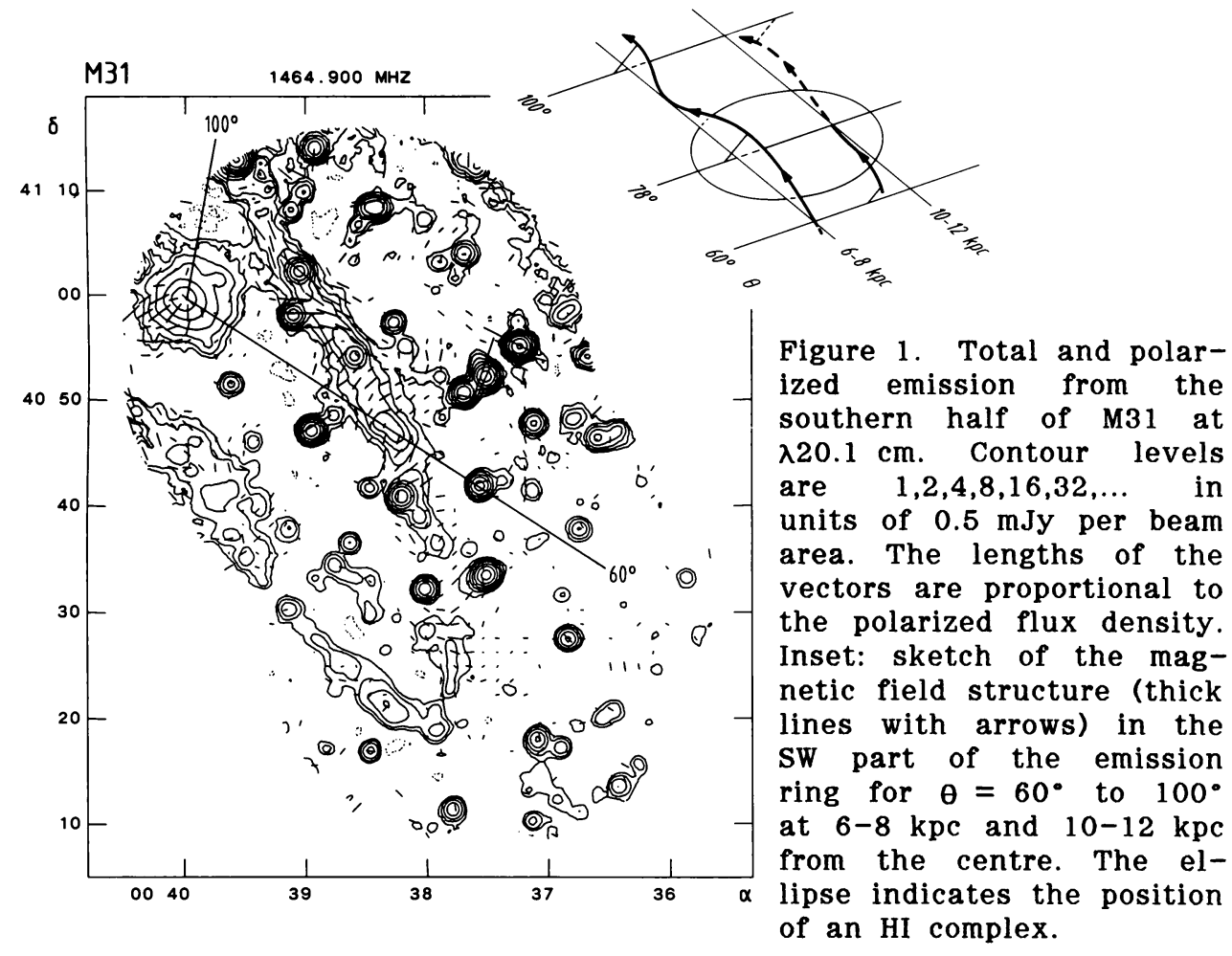


RUZMAIKIN: Are the deviations from the uniform field really periodical or random?

BECK: The region we studied in detail is not much larger than one "wavelength" of the periodicity possibly existing in M31. Hence we have to extend our survey in order to find out whether the deviations we found are periodic along the azimuthal angle or not.

BROWNE: Could you interpret the spiral arm field as helical?

BECK: No; unless there is only one helical bundle of field lines within the telescope beam with a small inclination against the spiral arm, which seems unreasonable.

BROWNE: I think one helical bundle is reasonable if there is vorticity.

KULSRUD: Would the deviations in $\vec{B}$ produced by superbubbles (multiple supernovae) be observable in M31?

BECK: The linear resolution in the VLA survey of M31 is 250 pc so that field deviations by superbubbles could be observable. However, nothing like that is found in the sw quadrant. 